\title{
Creating Cartilage in Tissue-Engineered Chamber Using Platelet-Rich Plasma Without Cell Culture
}

\author{
Meishui Wang, MD, ${ }^{1, *}$ Guojie Chen, MD, ${ }^{1,2,{ }^{*}}$ Guanmin Li, MD, ${ }^{1}$ Biao Wang, MD, PhD, ${ }^{1}$ and Chen Lei, MD ${ }^{1}$
}

Background: Clinically available cartilage, such as large-volume tissue-engineered cartilage, is urgently required for various clinical applications. Tissue engineering chamber (TEC) models are a promising organlevel strategy for efficient enlargement of cells or tissues within the chamber. The conventional TEC technology is not suitable for cartilage culture, because it lacks the necessary chondrogenic growth factor, which is present in platelet-rich plasma (PRP). In this study, we added autogenous auricular cartilage fragments mixed with PRP in a TEC to obtain a large amount of engineered cartilage.

Experiment: To prove the efficacy of this method, 48 New Zealand white rabbits were randomly divided into 4 groups: PRP, vascularized (Ves), PRP, PRP+Ves, and control. Auricular cartilage was harvested from the rabbits, cut into fragments $(2 \mathrm{~mm})$, and then injected into TECs. Cartilage constructs were harvested at week 8 , and construct volumes were measured. Histological morphology, immunochemical staining, and mechanical strength were evaluated.

Results: At week 8, PRP+Ves constructs developed a white, cartilage-like appearance. The volume of cartilage increased by $600 \%$ the original volume from 0.30 to $1.8 \pm 0.1789 \mathrm{~mL}$. Histological staining showed proliferation of edge chondrocytes in the embedded cartilage in the PRP and PRP+Ves groups. Furthermore, the cartilage constructs in the PRP+Ves group show mechanical characteristics similar to those of normal cartilage.

Conclusions: Auricular cartilage fragments mixed with PRP and vascularization of the TEC showed a significantly increased cartilage tissue volume after 8 weeks of incubation in rabbits.

Keywords: tissue engineering chamber, axial vascular network, platelet-rich plasma, chondrogenesis

\section{Impact Statement}

Repair of defects of ear cartilage tissue has always been a huge challenge to plastic surgeons. In this article, a new method is presented to produce within 8 weeks auricular cartilage in a tissue engineering chamber without cell culture. Having such a method is a valuable step toward creating a large volume of functional cartilage tissue, which may lead to successful construction of normal auricular structure with minimal donor-site morbidity.

\section{Introduction}

$\mathbf{T}$ OTAL AND SUBTOTAL reconstruction of auricle defects remains challenging for plastic surgeons. Some surgeons have reported using rib cartilage, the current golden standard, to carve a neo-framework, resulting in aesthetically acceptable results. ${ }^{1,2}$ However, this procedure is inevitably associated with donor site morbidity. To address this issue, the field of cartilage tissue engineering has emerged in the last 30 years. For example, in 1997, Cao et al. engineered car- tilage using a human auricle shape implanted into the back of nude mice; since then, many researchers have explored strategies for constructing tissue-engineered cartilage.

Cartilage is a type of avascular tissue, and chondrocytes were expected to be easily cultured in vitro. ${ }^{4}$ However, although isolated chondrocytes survive and proliferate robustly in vitro during cell culture, after several passages of monoculture, cells lose their natural phenotype and undergo degeneration. ${ }^{5,6}$ In contrast, mesenchymal stem cells (MSCs), including adipose-derived stem/stromal cells, show great

\footnotetext{
${ }^{1}$ Department of Plastic and Cosmetic Surgery, The First Affiliated Hospital of Fujian Medical University, Fuzhou, P.R. China.

${ }^{2}$ Department of Burn and Plastic Surgery, The Fourth Medical Centre, Chinese PLA General Hospital, Beijing, P.R. China.

*Both these authors contributed equally to this work.
} 
proliferation efficiency but exhibit a hypertrophic morphology after the induction of chondrogenic differentiation. Therefore, coculture strategies have been developed to circumvent some of the limitations of utilizing chondrocytes or MSCs alone. However, the risk of contamination during cell culture has limited the clinical use of this approach. ${ }^{7-9}$

Yanaga et al. generated clinically available cartilage stacking cultured monolayer chondrocytes into multilayer structure and using platelet-rich plasma (PRP) as a scaffold and implanted these multilayered chondrocyte constructs into the abdomen. Subsequently, after 6 months of implantation, a mature cartilage block was formed with sufficient elasticity for use as auricular cartilage. ${ }^{10}$ These findings demonstrated that multilayered chondrocyte constructs in combination with PRP can imitate the structure of cartilage, thereby promoting the regeneration of chondrocytes and consequently generating engineered cartilage bricks in vivo. In addition, Kawanabe and Nagata demonstrated that reembedding sliced rib cartilage into the empty pocket of the perichondrium can stimulate the regeneration of cartilage; 6-12 months were required to regenerate cartilage tissue in the perichondrium pockets. ${ }^{11}$ This finding suggests the possibility of using sliced cartilage as a cell source for cartilage tissue engineering.

As a follow-up of the above mentioned, organ-level tissue engineering strategies ${ }^{12}$ and tissue engineering chambers (TECs $)^{13}$ were introduced to produce a clinically relevant amount of cartilage. However, these culture techniques still take too long and therefore lacks clinical application value. By introducing an intrinsic vascular component into a rigid chamber, TECs can stimulate the regeneration of cells ${ }^{14}$ or tissues $^{15,16}$ in the chamber. Our team previously used TECs to develop adipose flaps with a volume of over $80 \mathrm{~mL}$ in rabbits. ${ }^{17}$

Consequently, PRP, which contains both chondrogenic and angiogenic factors, was introduced by us into the TEC as a method to optimize this technology for the production of a large amount of viable engineered cartilage.

\section{Methods}

Rectangular-shaped TECs, made of silicone rubber, were used. They had an inner length of $20 \mathrm{~mm}$, forming a $3.2-\mathrm{mL}$ wide space with a height of $8 \mathrm{~mm}$. All chambers were per- forated with side holes measuring $1 \mathrm{~mm}$ in diameter. The base had four suture holes for fixation, allowing the chamber to be anchored to the surrounding tissues.

Rabbits were used, as experimental animal, and anesthetized with $30 \mathrm{mg} / \mathrm{kg}$ of $3 \%$ pentobarbital. Lidocaine was applied to the operative area by multipuncture of $0.5 \mathrm{~mL} /$ site. The surgery process is shown in Figure 1. First, a $1 \times 1 \mathrm{~cm}$ portion of auricular cartilage was harvested from both ears of a rabbit. The $1.5-\mathrm{cm}$ incision was closed immediately using nylon sutures. The harvested cartilage was cut using a lancet into $1-2-\mathrm{mm}^{3}$ blocks. Then, a moderate amount $(10 \mathrm{~mL})$ of whole blood was drawn through the marginal ear vein and centrifuged to prepare PRP, as reported by $\mathrm{Wu}$ et al., ${ }^{18}$ and immediately transferred to sterile tubes containing heparin. Samples were centrifuged (Eppendorf) for $10 \mathrm{~min}$ at $215 \mathrm{~g}$, resulting in three layers. Plasma layers were then removed and subjected to another 10-min centrifugation at $863 g$ to obtain platelet-poor plasma (PPP) and PRP. The PPP was discarded, while thrombin was added to the PRP to change it into a hydrogel state. Finally, PRP and diced cartilage were mixed and loaded into a $1-\mathrm{mL}$ syringe.

Subsequently, a 3-cm incision was made in the skin of the inguinal area. A subcutaneous pocket was created around the incision, and the femoral artery bundles were recognized and isolated. Then, the perforated TEC was installed and fixed using 3-0 nylon for the isolated artery. Subsequently, cartilage with PRP was injected into the chamber through the base of the chamber. Finally, the wound was closed with 4-0 nylon. Postoperatively, penicillin was administered intramuscularly to prevent infection. After 8 weeks of implantation, rabbits were euthanized and the cartilage was harvested for further analysis.

\section{Experiment}

\section{Experimental design}

Animals. Forty-eight New Zealand white rabbits (weight: 2.5-3.0 kg, age: 3-4 months) were used in this study. All rabbits were purchased and housed at the Experimental Animal Center of Fujian Medical University. All experiments were performed with the approval of the Ethics Committee of our hospital and the Experimental Animal Center of Fujian Medical University (FJMU IACUC2018-0002).
FIG. 1. Schematic diagram of the experimental model of cartilage tissue engineering chamber. Left: the vessels we used and the surgical area. (A) Cartilage Group. (B) Cartilage+Vessel. (C) Cartilage and PRP. (D) Cartilage, PRP, and Vessel. PRP, platelet-rich plasma. Color images are available online.
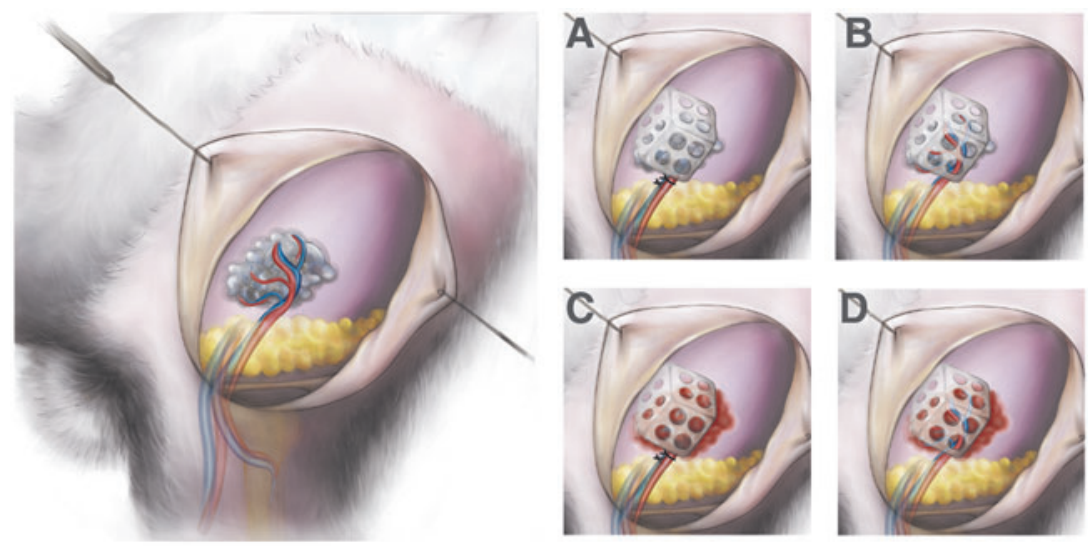

Chamber 
After anesthesia, auricular cartilage of the ears of the rabbits was dissected and PRP was prepared and loaded with cartilage fragments according to the description in the Methods section. Then, subcutaneous pockets were created in the inguinal area and TECs installed. Subsequently, cartilage with or without PRP was injected into the chamber through the base of the chamber. Therefore, the rabbits were randomly divided into four groups as shown in Figure 1: (1) PRP group $(1.0 \mathrm{~mL}$ PRP was mixed with $0.30 \mathrm{~mL}$ cartilage fragments and injected into the TEC), (2) Ves group (the femoral artery was dissected and left in situ in the TEC, which was injected cartilage fragments only), (3) PRP+Ves group (the femoral artery was dissected and left in situ in the TEC, which was injected with cartilage fragments mixed with PRP), and (4) Control group (cartilage fragments only were injected into the TEC).

Finally, the wounds were closed with 4-0 nylon. Postoperatively, penicillin was administered intramuscularly to prevent infection.

Tissue harvesting. Animals were euthanized at 8 weeks. Constructs were harvested and carefully removed from chambers via separation from the surrounding tissue. The volumes were measured by drainage after imaging with a digital camera. Harvested cartilage was placed in a $5 \mathrm{~mL}$ syringe, to which $3 \mathrm{~mL}$ saline has been added in advance. The volume measured by the syringe minus $3 \mathrm{~mL}$ is the cartilage volume. All samples were prepared for subsequent analyses.

Histological examination. All samples were fixed in 4\% paraformaldehyde, dehydrated, and embedded in paraffin. Specimens were then sectioned serially ( $5-\mu \mathrm{m}$ thickness) along the longitudinal axis. The sections were stained with hematoxylin and eosin (H\&E), safranin-O, Verhoeff-Van Gieson, and Masson's trichrome stains or immunostained for Ki-67. Sections stained with H\&E were used to determine chondrocyte viability, chondroid tissue status, and the structure of the surrounding tissues, whereas sections stained with Masson's trichrome stain were used to determine the collagen content of the construct tissue matrix (green color). Safranin-O staining was used to determine the proteoglycan content of the matrix (red color). Sections stained with Verhoeff-Van Gieson were used to assess chondroid tissue matrix metachromasia and the density of vessels. Ki-67 immunohistochemistry was used to identify the locations of chondrocyte proliferation in the specimen (brown deposits in the nucleus). In brief, the sections were treated with 0.3 vol\% hydrogen peroxide in methanol solution, and blocked with $1 \mathrm{wt} \%$ bovine serum albumin in phosphate buffered saline for $30 \mathrm{~min}$. Then, primary antibodies (ab15580; Abcam) were incubated overnight at $4^{\circ} \mathrm{C}$. Following the incubation, the samples were treated with peroxidase-conjugated secondary antibody (ab6721; Abcam). The immunoreactions were visualized by $\mathrm{DAB}$ substrate in chromogen solution. The samples were counter stained with hematoxylin. All sections were assessed by microscopy (Olympus).

Histopathological analysis. The degree of cartilage fibrosis was calculated as a percentage of the stained area (not including the capsule area) divided by the total tissue area of the sections using ImageJ software (National Institutes of Health, Bethesda, MD). The microvessel density of the capsule was calculated as a percentage of the stained microvessel area divided by the total tissue area of the sections using ImageJ software and we took the five random areas with the highest blood vessel density section under the microscope, and express the microvessel density as the ratio of the area occupied by blood vessels to the visual field area. Cartilage-specific extracellular matrix (ECM) was calculated as a percentage of the stained area (Safranin-O staining and Verhoeff-Van Gieson staining) divided by the total tissue area of the sections using ImageJ. Cell proliferation was calculated as a percentage of the $\mathrm{Ki}-67$ positive cells divided by the total cell number of the sections using ImageJ software.

Mechanical test. The biomechanical properties of the engineered cartilage were determined using a texture analyzer (Stable Micro System). The samples were processed to a rectangular shape by means of refinement; cylindrical samples had a 5-mm side length and $2 \mathrm{~mm}$ thickness. A constant compressive strain rate of $2.0 \mathrm{~mm} / \mathrm{s}$ was applied until $80 \%$ of the maximum deformation was achieved, and a stress-strain curve was generated. The Young's modulus was calculated based on the slope of the stress-strain curve.

\section{Statistical analysis}

All quantitative data were expressed as means \pm standard deviations. Statistical analyses were carried out with GraphPad Prism 7.0 software. Comparisons between groups were performed by random data two-way analysis of variance. Significance was established for differences with $p$-values of $<0.05$.

\section{Experimental Results}

\section{Macroscopic observations}

At the end of 8 weeks, every animal developed a vascularized double-layered fibrous capsule around the implanted engineered cartilage and the implanted chambers. Only the constructs in the PRP+Ves group filled the chamber and were shaped by the chamber into a square cube. The diced cartilage formed an integral tissue in all groups (Fig. 2). However, from the cross-section view, we could distinguish clear borders of the implanted cartilage in the control group. In contrast, the borders in the PRP and PRP+Ves groups were blurred compared with that in the control group. The femoral vessels in the Ves and PRP+Ves groups, which had been transplanted and fixed in the chamber, were fully incorporated into the capsule on the constructed tissue surface (Fig. 3A).

\section{Cartilage volume increased in all groups}

The initial volume of cartilage was $0.30 \mathrm{~mL}$ in each group. The volumes of all constructs were significantly higher than their initial volumes $(p<0.05)$. The volume in the PRP+Ves group increased by $600 \%$ to $1.80 \pm 0.18 \mathrm{~mL}$, and this volume was significantly higher than those in the other three groups (Fig. 4; $p<0.05$ ). Moreover, the volumes in the Ves and PRP groups increased to $1.25 \pm 0.15$ and $1.26 \pm$ $0.21 \mathrm{~mL}$, respectively. Overall, the volumes in all experimental groups were significantly higher than that in the control group. However, there were no differences between the Ves and PRP groups in terms of the volume of engineered cartilage ( $p>0.05$; Fig. 4A). 
FIG. 2. Tissue-engineered cartilage in the group of control, Vessel, PRP, and $\mathrm{PRP}+$ Ves under gross view and cross section. Color images are available online.
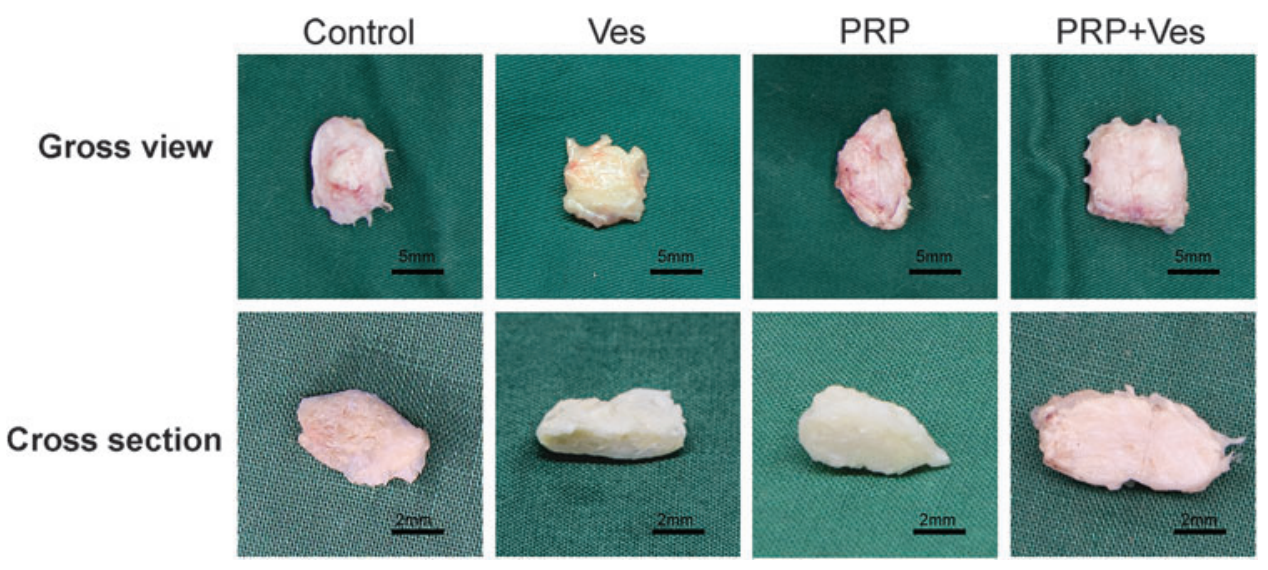

Hematoxylin and eosin and Masson's trichrome staining of cartilage constructs

In cartilage sections for all groups, chondrocytes could be easily distinguished within the matrix. In the control and Ves groups, the embedded cartilage had clear borders by
H\&E staining. The original cartilage showed darker purple staining for the ECM, and the surrounding connective tissue was stained pink. Moreover, the border of original cartilage in the PRP and PRP+Ves group was blurred. On the border, the chondrocytes and collagen interdigitate with each other.
A

\section{Grossview}

$\mathrm{HE}$

FIG. 3. (A) Assessment of vascularized capsule tissue surrounding the engineered cartilage gross view of vascularized capsule tissue. The femoral vessels that had been transposed into the chamber was well preserved (marked as black arrow). Histologic analysis of capsule tissue in each group after 8 weeks in vivo. Capillary structure were observed in all groups (marked as “*”). Scale bars = $100 \mu \mathrm{m}$. (B) The microvessel density of the capsule was accessed by ImageJ software among the groups. Error bars represent mean $\pm \mathrm{SD}$ for $n=6$. *Significantly different $(p<0.05)$ values among the experimental treatments. Color images are available online.

Van-Gieson

Masson
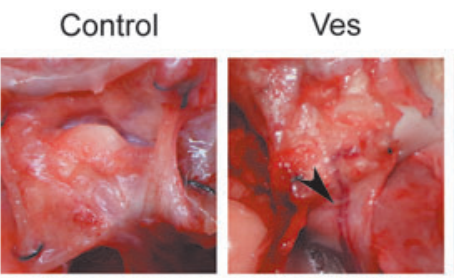

PRP

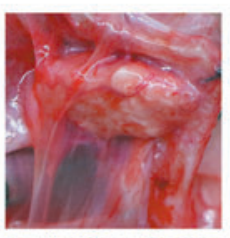

$\mathrm{PRP}+\mathrm{Ves}$
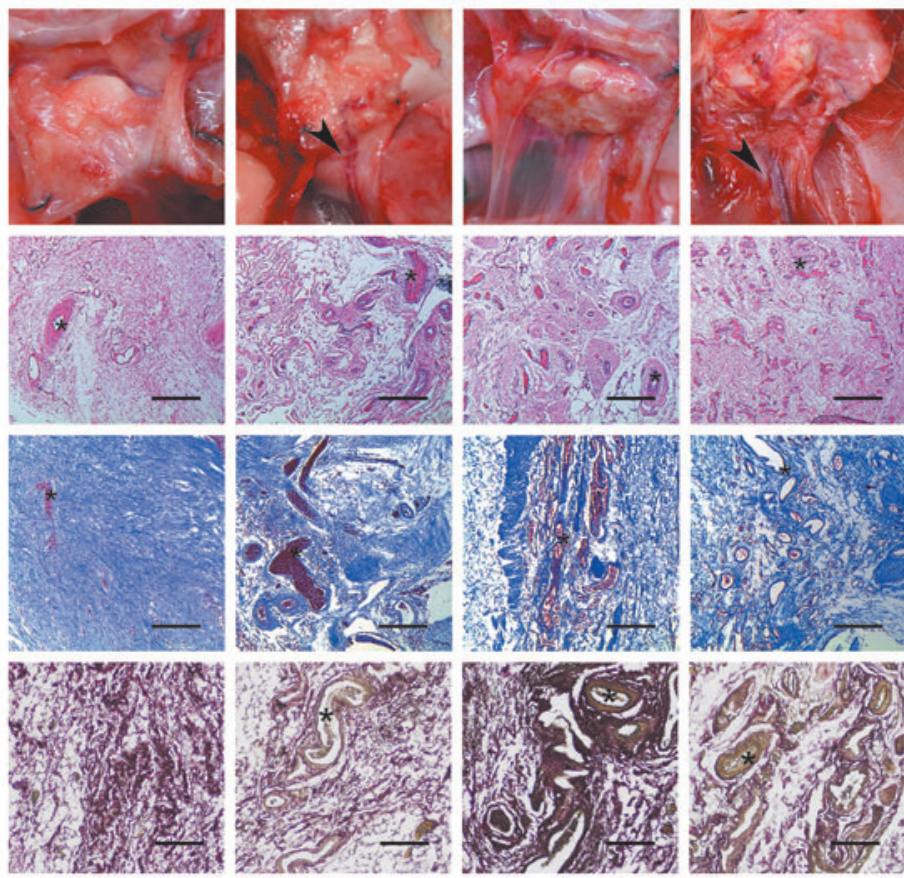

B

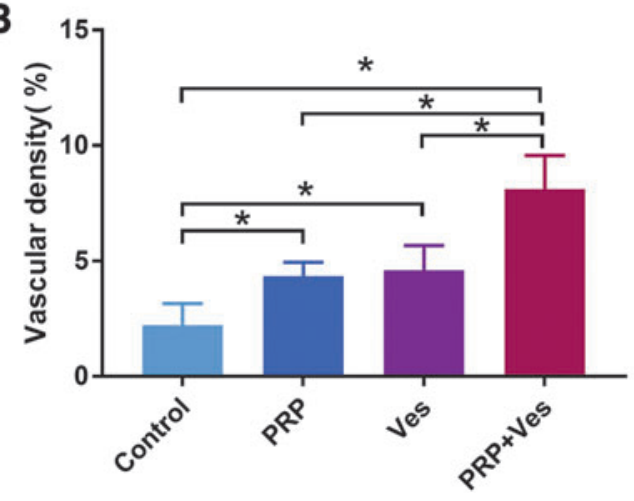

- Control

- PRP

- Ves

- PRP+Ves 


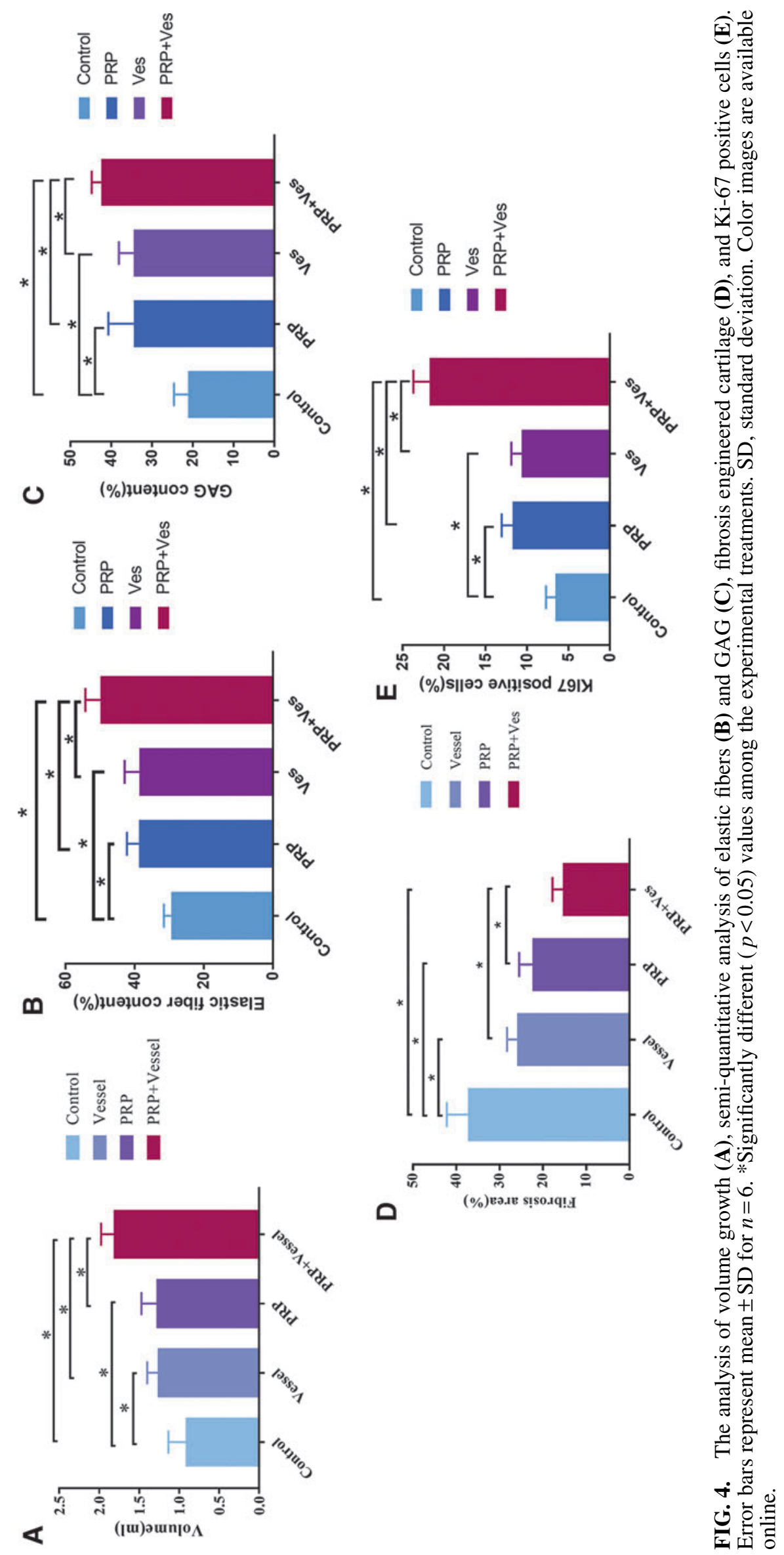


The different merging degree of the embedded cartilage and neo-tissue in all groups was confirmed by Masson's trichrome stain. Furthermore, we quantified the fibrous component according to Masson's trichrome stain using ImageJ (Fig. 4D). The control group had the highest degree of cartilage fibrosis, whereas the PRP+Ves group showed the lowest degree of fibrosis $(15.02 \% \pm 1.24 \%)$. Indeed, the control, Ves, and PRP groups showed increased blue staining $(36.94 \% \pm 2.37 \%$, $25.61 \% \pm 1.20 \%$, and $22.05 \% \pm 1.56 \%$, respectively; $p<0.05$ ) compared with that in the PRP+Ves group.

\section{Safranin-O and Verhoeff-Van Gieson staining of auricle cartilage-specific extracellular matrix components}

Both Safranin-O and Verhoeff-Van Gieson staining showed strong positive signals in the embedded cartilage matrix. The border of embedded cartilage and neo-tissue was clear in the control and Ves groups. Interestingly, both stains faded on the periphery of the cartilage in the PRP and $\mathrm{PRP}+\mathrm{Ves}$ groups. We quantified the glycosaminoglycans and elastic fibers according to Safranin-O and Verhoeff-Van Gieson staining using ImageJ (Fig. 4C, D). The PRP+Ves group showed the highest degree of GAG content $(42.48 \% \pm$ $2.23 \%$ ). The control, Ves, and PRP groups showed decreased GAG content $(21.17 \% \pm 1.55 \%, 34.5 \% \pm 1.63 \%$, and $34.49 \% \pm 2.78 \%$, respectively; $p<0.05$ ) compared with that in the PRP+Ves group. Verhoeff-Van Gieson staining shows similar results, The $\mathrm{PRP}+\mathrm{Ves}$ group showed the highest degree of elastin content $(49.92 \% \pm 1.95 \%)$. The control, Ves, and PRP groups showed decreased GAG content $(29.42 \% \pm 0.96 \%$,
$38.66 \% \pm 1.89 \%$, and $38.79 \% \pm 1.56 \%$, respectively; $p<0.05$ ) compared with that in the PRP+Ves group.

The stainless ECM around the chondrocytes on the periphery indicated that these chondrocytes may be newly formed.

\section{Ki-67 positive staining on the periphery of embedded cartilage revealed an outgrowth phenomenon}

Ki-67 staining (Fig. 5) was positive on the periphery of embedded cartilage, demonstrating robust proliferation of chondrocytes in all groups except for the control group. Thus, Ki-67 immunohistochemical staining on the periphery revealed an outgrowth phenomenon, whereas the chondrocytes in the center showed negative staining. We quantified the Ki67 positive cells using ImageJ (Fig. 4E). The PRP+Ves group has the highest amount of Ki-67 positive cells $(21.75 \% \pm$ $0.88 \%$ ). The control, Ves, and PRP groups showed relatively small number of Ki-67 positive cells $(6.59 \% \pm 0.50 \%$, $10.65 \% \pm 0.56 \%$, and $11.76 \% \pm 0.59 \%$, respectively; $p<0.05$ ) compared with that in the PRP+Ves group.

\section{The fibrous capsule wrapping the cartilage showed vascularization}

Capillary structures were more prominent in the Ves, $\mathrm{PRP}$, and PRP+Ves groups than in the control group (Fig. 3A). ImageJ analysis showed that the microvessel density in the PRP+Ves group was significantly increased to $\sim 7.31 \% \pm 0.92 \%$, which was higher than those in the control, Ves, and PRP groups $(2.41 \% \pm 0.63 \%, 4.35 \% \pm$
FIG. 5. Histologic and immunohistochemical analysis of engineered cartilage tissue in each group after 8 weeks in vivo. The boundary of original cartilage block is shown by the dotted. Neocartilage formation (marked as “*”) is remarkable in $\mathrm{P}+\mathrm{V}$ group. Scale bars $=100 \mu \mathrm{m}$. Color images are available online.

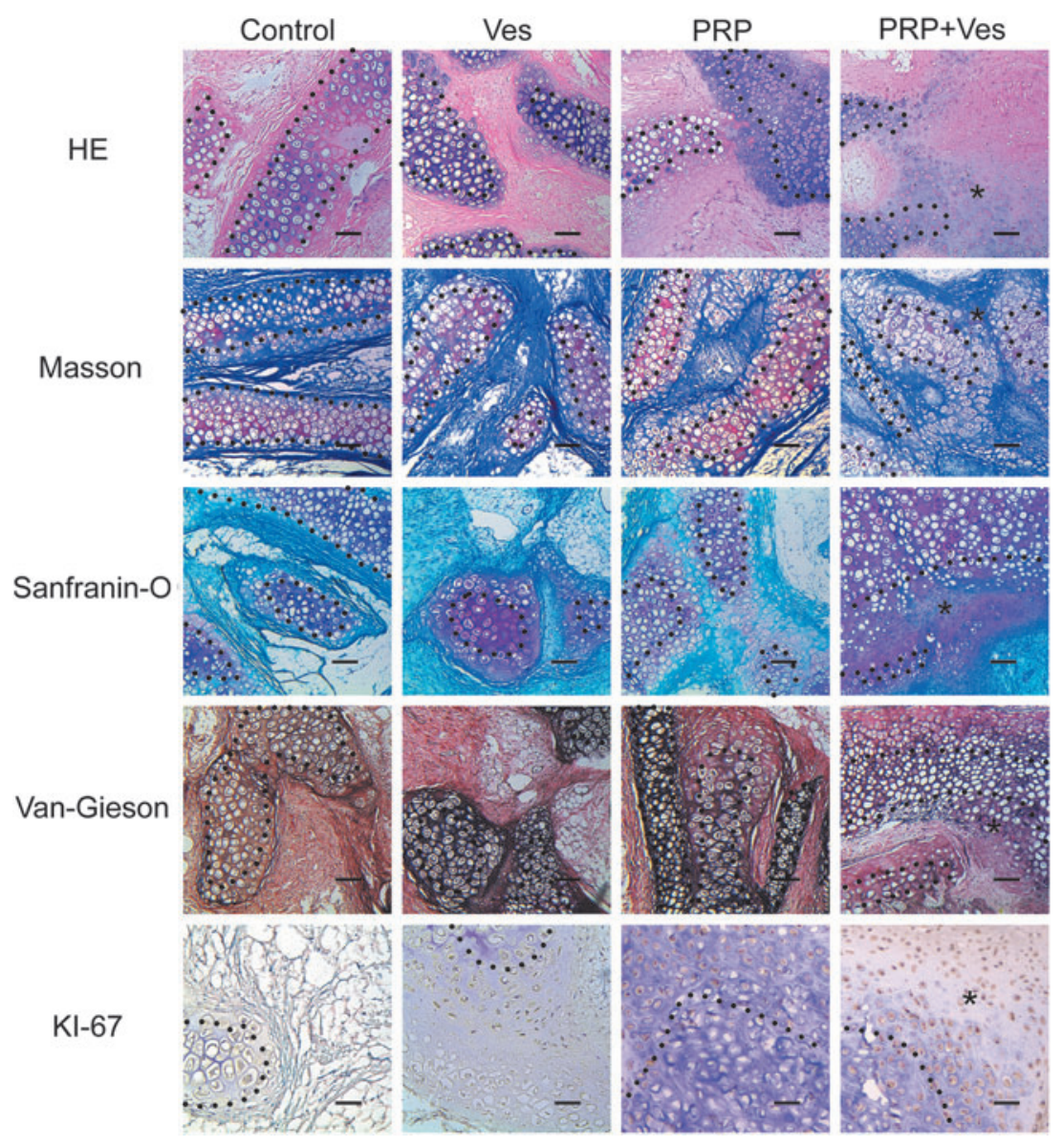


$0.48 \%$, and $4.69 \% \pm 0.64 \%$, respectively; $p<0.05)$. Moreover, the microvessel densities in the Ves and PRP groups were significantly higher than that in the control group $(p<0.05)$. There were no significant differences between the Ves and PRP groups (Fig. 3B).

\section{Biomechanical analyses}

To further quantitatively evaluate the cartilage-like properties of the engineered constructs, biomechanical analyses were performed. The Young's modulus for samples in the Ves and PRP+Ves groups did not differ significantly from those in normal cartilage. The Young's moduli in the control and PRP groups were significantly lower than that in normal cartilage (Fig. 6B).

\section{Discussion}

Cartilage tissue engineering has recently emerged as a novel medical technology, and continuous efforts have been made to establish ear-shaped cartilage tissue engineering. ${ }^{2}$ However, limited strategies are available for achieving clinically viable auricle reconstruction. ${ }^{17,19}$ One of the major challenges in such tissue engineering is obtaining a sufficient cell source. For cartilage tissue engineering, chondrocyte monolayer cultures tend to dedifferentiate or degenerate, ${ }^{6}$ change phenotypically, and show altered ECM secretion. ${ }^{20}$ Furthermore, chondrocytes from microtia cartilage showed inferior capacity to yield healthy ear chondrocytes. ${ }^{21}$ Although many attempts have been made to slow dedifferentiation during passaging, including the use of hypoxic conditions, ${ }^{22}$ threedimensional (3D) bioscaffolds, ${ }^{23}$ high-density culture, ${ }^{24}$ growth factors, ${ }^{25}$ and varying temperatures, ${ }^{24}$ no efficient methods have been developed to overcome these problems. ${ }^{20}$ Alternatively, MSCs may represent a promising cell source owing to the chondrogenic differentiation ability of these cells. ${ }^{26}$ Chondrocytes obtained from MSC differentiation show hypertrophic morphology and expression of collagen $X^{4,27}$ Although strategies for coculture of MSCs and chondrocytes have been developed ${ }^{1}$ to overcome the drawbacks of single cells alone, the complex procedures have made clinical use difficult owing to the necessity for optimization of coculture conditions and for increased product stability. ${ }^{7}$

The regeneration potential of chondrocytes alone is sufficient for ear-cartilage tissue engineering. ${ }^{10}$ Indeed, researchers have developed a two-stage strategy for ear reconstruction involving incubation of cartilage blocks. Yanaga used a multilayer culture technique involving reseeding of chondrocytes onto dedifferentiated chondrocytes under monolayer culture, causing the chondrocytes to redifferentiate again. Thus, such a multilayer structure resembling the natural structure of cartilage is crucial for the regeneration of chondrocytes. According to the study by Yanaga et al., we used auricle cartilage fragments, which maintained the natural structure of cartilage. Finally, we generated a cartilage block with a volume of $\sim 2 \mathrm{~mL}$, which was about $600 \%$ the original volume. Histological assessment using Ki-67 staining confirmed an edge-outgrowth phenomenon. Furthermore, we found that the neo-cartilage was positive for both Safranin-O and Verhoeff-Van Gieson staining, demonstrating the auricle cartilage origin of the tissue.

After physically cutting the cartilage, small pieces of cartilage show outgrowth at the cutting edge in vivo ${ }^{28}$ and in vitro. ${ }^{29,30}$ Although some researchers have shown limited outgrowth, ${ }^{31,32}$ no researchers have used scaffolds to optimize regeneration. Additionally, the reported observation times have always been $<7$ days, which is not sufficient for outgrowth. The regeneration of cartilage fragments may take more than 6 months, and the remodeling of regenerated cartilage is even sustained until 12 months, as demonstrated in a clinical study. ${ }^{11}$ Moreover, the scaffold used for cartilage is a crucial factor determining the results of regeneration. ${ }^{33,34}$

In our study, we used PRP as the scaffold to carry cartilage fragments. PRP is an autologous extract that has low immunogenicity and some anti-inflammatory effects. ${ }^{35} \mathrm{Re}$ cently, many studies have used PRP alone or in combination with other scaffold materials in cartilage tissue engineering. ${ }^{35-38}$ PRP is a carrier of multiple cytokines, PDGF, IGF-1, and FGF among them promoting the regeneration of chondrocytes and VEGF, TGF-1 and PDGF-BB promoting angiogenesis. $^{39,40}$ Additionally, PRP forms a 3D porous fibrin structure, allowing diffusion of the surrounding components and supporting the differentiation of chondrocytes. ${ }^{38}$ By using PRP as a scaffold, Yanaga et al. produced clinically applicable cartilage blocks. ${ }^{10}$ Thus, we believe that PRP may be an ideal bioactive scaffold for cartilage tissue engineering.

In the PRP+Ves group, we observed merging of the neocartilage with the embedded cartilage. In the clinical setting, this merging is observed by about 10-12 months. ${ }^{11}$ Thus, in $\mathrm{PRP}+\mathrm{Ves}$ group, the regeneration process was accelerated. In our study, we used a TEC technique that introduced an intrinsic vessel into the constructs. ${ }^{41}$ Although cartilage is an avascular tissue that contains chondrocytes alone, the exchange of oxygen and nutrients is required during regeneration. ${ }^{42}$ In fact, the
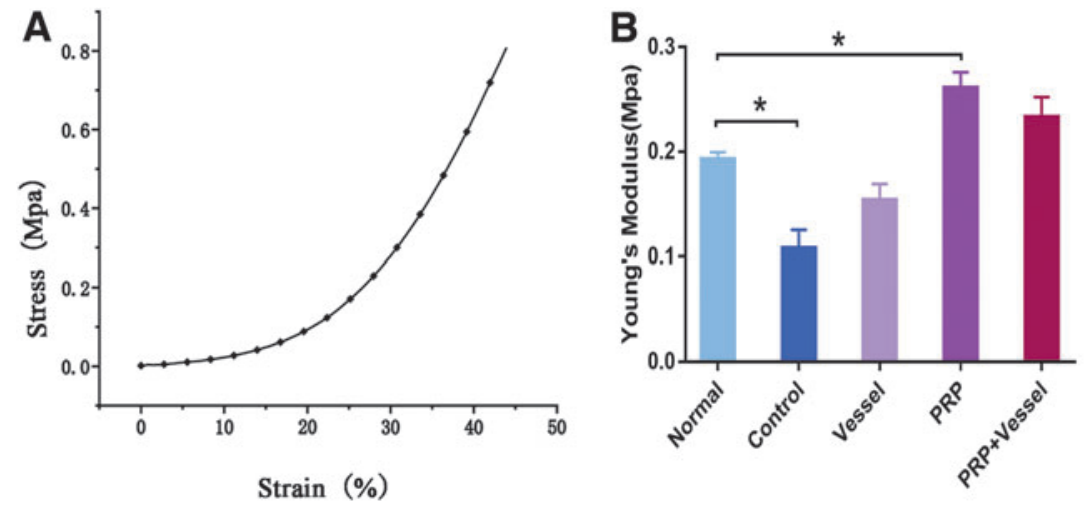

FIG. 6. (A) Stress-strain curve and (B) compressive Young's modulus among normal rabbit auricular cartilage and the group of control, Vessel, PRP, and PRP + Vessel after 8-week implantation. *Significant difference $(p<0.05)$ in the elastic modulus values between PRP group and the control group and normal rabbit auricular cartilage. Color images are available online. 
perichondrium is a well-vascularized tissue. Despite the observation that invasion of vessels into cartilage is a sign of aging, it is difficult to determine whether vessels can cause chondrocyte aging or whether hypotrophic chondrocytes cause the invasion of vessels. ${ }^{43}$ von Bomhard et al. showed that without introducing blood vessels, the center of an embedded ear-shaped scaffold contained no vital cells, but instead exhibited large areas of necrosis. ${ }^{44}$ Although we did not observe necrosis in our study, we believed that vessels are crucial for clinical applications of TECs. In the PRP group, we also observed that the capsule wrapping the cartilage was well vascularized. Indeed, PRP releases many proangiogenic cytokines, which could explain why the PRP group showed similar merging of cartilage fragments compared with the Ves and $\mathrm{PRP}+\mathrm{Ves}$ groups. Furthermore, intrinsic vessels only formed a vascular capsule wrapping the cartilage without invading into the cartilage, as demonstrated by our histological findings.

There were several limitations in this study of ours, the exact process of the regeneration of chondrocytes was not clearly demonstrated. According to the Ki-67 result on week 8 , the proliferation of chondrocytes on the outer layer of the embedded cartilage fragment was still robust, indicating that the regeneration process did not finish. Thus the long-term stability of the cartilage contruct was not clear. Furthermore, large animal study need to be done to fully explore the uplimit of contruct volume using TEC technique.

\section{Conclusion}

Using the TEC technique, we found that the addition of auricular cartilage fragments mixed with PRP and introduction of axial blood vessels promoted the growth of cartilage tissue, activated chondrocyte proliferation, and stimulated the secretion of cartilage-specific ECM. This approach enabled us to obtain clinically available tissue cartilage within a short time ( 8 weeks) while maintaining the morphology of the cartilage tissue.

\section{Acknowledgment}

We would like to acknowledge the help from Mr. Zhiming Zhang for the help with mechanical test.

\section{Disclosure Statement}

No competing financial interests exist.

\section{Funding Information}

This work was supported by the Natural Science Foundation of China (81801931), the Scientific Research Foundation of National Health Planning Scientific Research Foundation-Joint Research Projects of Fujian Provincial Health and Education (2019-WJ-09), the Special Supported Funds of Fujian Provincial Finance Department (BPB-WB2019), the Young and Middle-aged Key Scholar Program of Fujian Provincial Health Commission (2019-ZQN-58), the Fujian Provincial Natural Science Foundation Program (2018J01154, 2018J01163).

\section{References}

1. Morrison, R.J., Nasser, H.B., Kashlan, K.N., et al. Coculture of adipose-derived stem cells and chondrocytes on three-dimensionally printed bioscaffolds for craniofacial cartilage engineering. Laryngoscope 128, E251, 2018.
2. Bichara, D.A., O'Sullivan, N.A., Pomerantseva, I., et al. The tissue-engineered auricle: past, present, and future. Tissue Eng Part B Rev 18, 51, 2012.

3. Cao, Y., Vacanti, J.P., Paige, K.T., Upton, J., and Vacanti, C.A. Transplantation of chondrocytes utilizing a polymercell construct to produce tissue-engineered cartilage in the shape of a human ear. Plast Reconstr Surg 100, 297, 1997.

4. Armiento, A.R., Stoddart, M.J., Alini, M., and Eglin, D. Biomaterials for articular cartilage tissue engineering: learning from biology. Acta Biomater 65, 1, 2018.

5. Harris, J.D., Siston, R.A., Brophy, R.H., et al. Failures, reoperations, and complications after autologous chondrocyte implantation-a systematic review. Osteoarthritis Cartilage 19, 779, 2011.

6. Niemeyer, P., Salzmann, G.M., Hirschmuller, A., and Sudkamp, N.P. Factors that influence clinical outcome following autologous chondrocyte implantation for cartilage defects of the knee. Z Orthop Unfall 150, 83, 2012.

7. Zhang, Y., Guo, W., Wang, M., et al. Co-culture systemsbased strategies for articular cartilage tissue engineering. J Cell Physiol 233, 1940, 2018.

8. Dahlin, R.L., Meretoja, V.V., Ni, M., Kasper, F.K., and Mikos, A.G. Chondrogenic phenotype of articular chondrocytes in monoculture and co-culture with mesenchymal stem cells in flow perfusion. Tissue Eng Part A 20, 2883, 2014.

9. Mehta, S., Akhtar, S., Porter, R.M., Onnerfjord, P., and Bajpayee, A.G. Interleukin-1 receptor antagonist (IL-1Ra) is more effective in suppressing cytokine-induced catabolism in cartilage-synovium co-culture than in cartilage monoculture. Arthritis Res Ther 21, 238, 2019.

10. Yanaga, H., Imai, K., Fujimoto, T., and Yanaga, K. Generating ears from cultured autologous auricular chondrocytes by using two-stage implantation in treatment of microtia. Plast Reconstr Surg 124, 817, 2009.

11. Kawanabe, Y., and Nagata, S. A new method of costal cartilage harvest for total auricular reconstruction: part II. Evaluation and analysis of the regenerated costal cartilage. Plast Reconstr Surg 119, 308, 2007.

12. Rustad, K.C., Sorkin, M., Levi, B., Longaker, M.T., and Gurtner G.C. Strategies for organ level tissue engineering. Organogenesis 6, 151, 2010.

13. Zhan, W., Marre, D., Mitchell, G.M., Morrison, W.A., and Lim S.Y. Tissue engineering by intrinsic vascularization in an in vivo tissue engineering chamber. J Vis Exp 111, 54099, 2016.

14. Morritt, A.N., Bortolotto, S.K., Dilley, R.J., et al. Cardiac tissue engineering in an in vivo vascularized chamber. Circulation 115, 353, 2007.

15. Findlay, M.W., Dolderer, J.H., Trost, N., et al. Tissueengineered breast reconstruction: bridging the gap toward large-volume tissue engineering in humans. Plast Reconstr Surg 128, 1206, 2011.

16. Messina, A., Bortolotto, S.K., Cassell, O.C.S., Kelly, J., Abberton, K.M., and Morrison, W.A. Generation of a vascularized organoid using skeletal muscle as the inductive source. FASEB J 19, 1570, 2005.

17. Wan, J., Dong, Z., Lei, C., and Lu, F. Generating an engineered adipose tissue flap using an external suspension device. Plast Reconstr Surg 138, 109, 2016.

18. Wu, J., Huang, J.F., Qin, X.X., et al. Platelet-rich plasma inhibits Wnt/beta-catenin signaling in rabbit cartilage cells activated by IL-1beta. Int Immunopharmacol 55, 282, 2018.

19. Zhou, G., Jiang, H., Yin, Z., et al. In vitro regeneration of patient-specific ear-shaped cartilage and its first 
clinical application for auricular reconstruction. EBioMedicine 28, 287, 2018.

20. Nazempour, A., and Van Wie, B.J. Chondrocytes, mesenchymal stem cells, and their combination in articular cartilage regenerative medicine. Ann Biomed Eng 44, 1325, 2016.

21. Gu, Y., Kang, N., Dong, P., et al. Chondrocytes from congenital microtia possess an inferior capacity for in vivo cartilage regeneration to healthy ear chondrocytes. J Tissue Eng Regen Med 12, e1737, 2018.

22. Foldager, C.B., Munir, S., Ulrik-Vinther, M., Soballe, K., Bunger, C., and Lind, M. Validation of suitable house keeping genes for hypoxia-cultured human chondrocytes. BMC Mol Biol 10, 94, 2009.

23. Rampichova, M., Kost'akova Kuzelova, E., Filova, E., et al. Composite 3D printed scaffold with structured electrospun nanofibers promotes chondrocyte adhesion and infiltration. Cell Adh Migr 12, 271, 2018.

24. Schulze-Tanzil, G., de Souza, P., Villegas Castrejon, H., et al. Redifferentiation of dedifferentiated human chondrocytes in high-density cultures. Cell Tissue Res 308, 371, 2002.

25. Rakic, R., Bourdon, B., Hervieu, M., et al. RNA interference and BMP-2 stimulation allows equine chondrocytes redifferentiation in 3D-hypoxia cell culture model: application for matrix-induced autologous chondrocyte implantation. Int J Mol Sci 18, 1842, 2017.

26. Barry, F., Boynton, R.E., Liu, B., and Murphy J.M. Chondrogenic differentiation of mesenchymal stem cells from bone marrow: differentiation-dependent gene expression of matrix components. Exp Cell Res 268, 189, 2001.

27. Li, H., Huang, L., Xie, Q., et al. Study on the effects of gradient mechanical pressures on the proliferation, apoptosis, chondrogenesis and hypertrophy of mandibular condylar chondrocytes in vitro. Arch Oral Biol 73, 186, 2017.

28. Marmotti, A., Bruzzone, M., Bonasia, D.E., et al. Autologous cartilage fragments in a composite scaffold for one stage osteochondral repair in a goat model. Eur Cell Mater 26, 15, 2013.

29. Sage, A., Chang, A.A., Schumacher, B.L., Sah, R.L., and Watson, D. Cartilage outgrowth in fibrin scaffolds. Am J Rhinol Allergy 23, 486, 2009.

30. Ng, S.S., Su, K., Li, C., Chan-Park, M.B., Wang, D.A., and Chan, V. Biomechanical study of the edge outgrowth phenomenon of encapsulated chondrocytic isogenous groups in the surface layer of hydrogel scaffolds for cartilage tissue engineering. Acta Biomater 8, 244, 2012.

31. Zingler, C., Carl, H.D., Swoboda, B., Krinner, S., Hennig, F., and Gelse, K. Limited evidence of chondrocyte outgrowth from adult human articular cartilage. Osteoarthritis Cartilage 24, 124, 2016.

32. Gelse, K., Riedel, D., Pachowsky, M., Hennig, F.F., Trattnig, S., and Welsch, G.H. Limited integrative repair capacity of native cartilage autografts within cartilage defects in a sheep model. J Orthop Res 33, 390, 2015.

33. Cheng, A., Schwartz, Z., Kahn, A., et al. Advances in porous scaffold design for bone and cartilage tissue engineering and regeneration. Tissue Eng Part B Rev 25, 14, 2019.

34. Fu, N., Dong, T., Meng, A., Meng, Z., Zhu, B., and Lin, Y. Research progress of the types and preparation techniques of scaffold materials in cartilage tissue engineering. Curr Stem Cell Res Ther 13, 583, 2018.

35. Lee, H.R., Park, K.M., Joung, Y.K., Park, K.D., and Do, S.H. Platelet-rich plasma loaded in situ-formed hydrogel enhances hyaline cartilage regeneration by CB1 upregulation. J Biomed Mater Res A 100, 3099, 2012.

36. Beigi, M.H., Atefi, A., Ghanaei, H.R., Labbaf, S., Ejeian, F., and Nasr-Esfahani, M.H. Activated platelet-rich plasma improves cartilage regeneration using adipose stem cells encapsulated in a 3D alginate scaffold. J Tissue Eng Regen Med 12, 1327, 2018.

37. Wang, K., Li, J., Li, Z., et al. Chondrogenic progenitor cells exhibit superiority over mesenchymal stem cells and chondrocytes in platelet-rich plasma scaffold-based cartilage regeneration. Am J Sports Med 47, 2200, 2019.

38. Vinod, E., Vinod Francis, D., Manickam Amirtham, S., Sathishkumar, S., and Boopalan, P. Allogeneic platelet rich plasma serves as a scaffold for articular cartilage derived chondroprogenitors. Tissue Cell 56, 107, 2019.

39. Chang, N.J., Erdenekhuyag, Y., Chou, P.H., Chu, C.J., Lin, C.C., and Shie, M.Y. Therapeutic effects of the addition of platelet-rich plasma to bioimplants and early rehabilitation exercise on articular cartilage repair. Am J Sports Med 46, 2232, 2018.

40. Kurita, J., Miyamoto, M., Ishii, Y., et al. Enhanced vascularization by controlled release of platelet-rich plasma impregnated in biodegradable gelatin hydrogel. Ann Thorac Surg 92, 837, 2011.

41. Lei, C., Dong, Z., Wan, J., et al. Transferring the exudate in the tissue engineering chamber as a trigger to incubate large amount adipose tissue in remote area. J Tissue Eng Regen Med 12, e1549, 2018.

42. Neumeister, M.W., Wu, T., and Chambers, C. Vascularized tissue-engineered ears. Plast Reconstr Surg 117, 116, 2006.

43. Colnot, C., Lu, C., Hu, D., and Helms, J.A. Distinguishing the contributions of the perichondrium, cartilage, and vascular endothelium to skeletal development. Dev Biol 269, 55, 2004.

44. von Bomhard, A., Veit, J., Bermueller, C., et al. Prefabrication of 3D cartilage contructs: towards a tissue engineered auricle — a model tested in rabbits. PLoS One 8, e71667, 2013.

Address correspondence to: Biao Wang, MD, PhD

Department of Plastic and Cosmetic Surgery The First Affiliated Hospital of Fujian Medical University 20 Chazhong Road Fuzhou 350005, Fujian P.R. China

E-mail: 1812166371@qq.com

Chen Lei, MD

Department of Plastic and Cosmetic Surgery The First Affiliated Hospital of Fujian Medical University 20 Chazhong Road Fuzhou 350005, Fujian P.R. China

E-mail: 1702611@qq.com

Received: February 14, 2020

Accepted: June 8, 2020

Online Publication Date: June 14, 2020 